\title{
Hypoxia/reoxygenation causes inflammatory response in transgenic sickle mice but not in normal mice
}

\author{
D.K. Kaul ${ }^{1}$ and R.P. Hebbel ${ }^{2}$ \\ ${ }^{1}$ Albert Einstein College of Medicine, Bronx, New York, USA \\ ${ }^{2}$ University of Minnesota Medical School, Minneapolis, Minnesota, USA \\ This work was presented in part at Experimental Biology '99, Washington, DC, USA, in April, 1999, \\ and at the 41st Annual Meeting of the American Society of Hematology, New Orleans, Louisiana, USA, \\ in December, 1999. Portions of this work have appeared in abstract form (1999, FASEB J. 13:A178. 1999, Blood. 94:676a.).
}

Address correspondence to: Dhananjaya K. Kaul, Albert Einstein College of Medicine, 1300 Morris Park Avenue, Bronx, New York 10461, USA. Phone: (718) 430-3702; Fax: (718) 430-8969; E-mail: kaul@aecom.yu.edu.

Received for publication December 21, 1999, and accepted in revised form June 14, 2000.

In sickle cell anemia, the initiation, progression, and resolution of a vasoocclusive episode may present features of ischemia-reperfusion injury, with recurrent episodes of ischemia/hypoxia and reoxygenation promoting inflammation. Here, we have tested the hypothesis that hypoxia/reoxygenation triggers inflammation in the transgenic sickle mouse. In these mice, even at ambient air, peripheral leukocyte counts are elevated by 1.7 -fold and neutrophil counts by almost 3 -fold. Two hours of hypoxia, followed by reoxygenation, induced a greater than normal rolling flux and adhesion of leukocytes in these mice, but no leukocyte extravasation. When 3 hours of hypoxia was followed by reoxygenation, sickle mice, but not normal mice, showed a distinct inflammatory response characterized by an increased number of adherent and emigrated leukocytes. Because these events, which are exaggerated in sickle mice, are not seen in response to hypoxia alone, we conclude that they represent a form of reperfusion injury. Studies using an $\mathrm{H}_{2} \mathrm{O}_{2}$-sensitive probe revealed clear evidence of oxidant production in vascular endothelial cells after hypoxia/reoxygenation in sickle mice. Infusion of an anti-P-selectin antibody, but not an anti-E-selectin antibody, completely inhibited this inflammatory response and significantly increased wall shear rates. These findings suggest that leukocyte-endothelium interaction contribute to vasoocclusive events in the sickle mice and perhaps in human sickle disease.

J. Clin. Invest. 106:411-420 (2000).

\section{Introduction}

Sickle cell anemia is characterized by recurring acute vasoocclusive episodes and chronic damage to multiple organs. The pathogenesis of sickle cell anemia is due to a single point mutation that results in the substitution of valine for glutamic acid at sixth position of the $\beta$ chain of the hemoglobin $\mathrm{S}(\mathrm{HbS})$ molecule. This single point mutation results in the polymerization of $\mathrm{HbS}$ and sickling of red cells under deoxygenated conditions. Although HbS polymerization is central to the pathophysiology of the disease, multiple factors may participate in the initiation of a vasoocclusive episode $(1,2)$.

In sickle cell anemia, at least two factors would contribute persistently to the vascular pathology. These two factors are sickling (oxy-deoxy cycles) and red-cell adhesion to endothelium, either of which can damage endothelium $(1,2)$. In addition, the initiation, progression, and resolution of a vasoocclusive episode may present features common with "reperfusion injury." This term refers to vascular damage that is attributable to the reintroduction of molecular oxygen and consequent generation of oxygen radicals that occurs after an ischemic episode $(3,4)$. In sickle cell disease, subclinical vasoocclusive events involving a transient blockage of vascular beds by red cell sickling and adhesion may be very frequent. Repeated and random occurrences of such events would adversely affect vascular endothelial cell function and contribute to multiple organ damage. Such episodes of reperfusion injury would result in a proinflammatory state in sickle cell anemia.

Both reperfusion injury and the rheological insult by SS red cells may lead to endothelial damage (5) and endothelial cell detachment $(6,7)$, as reported for other ischemic diseases (8). Recent studies have demonstrated that circulating endothelial cells in patients with sickle cell anemia have an abnormally activated phenotype (9, 10). A proinflammatory condition in sickle cell anemia is further indicated by higher than normal leukocyte counts $(11,12)$, elevated cytokines (13), and an increase in soluble intercellular adhesion molecule-1 (ICAM-1) and vascular cell adhesion molecules-1 (VCAM-1) (14, 15). Another potent inflammatory agent, platelet-activating factor (PAF), that participates in leukocyteendothelium interactions is elevated in patients with sickle cell anemia (16). Enhanced SS red cell-endothelium interaction can induce oxidant stress in cultured endothelium, resulting in transendothelial migration of monocytes (17). Interestingly, in patients with sickle cell 
anemia, infections are often followed by the occurrence of a vasoocclusive crisis $(18,19)$. Despite the evidence for a proinflammatory condition in sickle cell anemia, and a causal relationship between infection and vasoocclusion, there has been no study to our knowledge that defines leukocyte flow dynamics under in vivo conditions in the sickle context. Because leukocytes are more rigid and have a larger volume than red cells, an increase in their numbers and their enhanced interaction with endothelium would adversely affect overall microvascular hemodynamics and vascular resistance.

Reperfusion injury is characterized by leukocyte recruitment resulting in tissue dysfunction in various organ systems including heart, skeletal muscle, lungs, intestine, and skin (20-24). Leukocyte-endothelium interaction involves initial rolling (repeated transient contacts) of leukocytes along the endothelial surface followed by their firm adhesion and diapedesis. The rolling is mediated by selectins expressed on activated (but not quiescent) endothelial cells (25-27). After leukocyte rolling is initiated on the activated endothelium, activation of leukocyte $\beta 2$ integrins (CD11/CD18) leads to leukocyte interaction with endothelial ligands such as ICAM-1 $(26,28)$ and thereby results in arrest and firm adhesion of leukocytes on endothelial surface.

In the present studies, we used a transgenic mouse model expressing human $\alpha$ - and $\beta^{\mathrm{S}}$-globins on a mouse $\beta$-major thalassemic background (" $\beta$ s mouse") to test the hypothesis that hypoxia-induced red cell sickling followed by reoxygenation would trigger an inflammatory response, as evidenced by leukocyte behavior resembling that of known, previously studied models of reperfusion injury. The present studies were designed to compare leukocyte flow dynamics in control and transgenic mice both under steady-state conditions and after hypoxia/reoxygenation, and to ascertain whether the inflammatory response is associated with generation of oxidants (i.e., $\mathrm{H}_{2} \mathrm{O}_{2}$ ) in vascular endothelium.

\section{Methods}

Transgenic mice. Transgenic sickle mice expressing human $\alpha\left(\alpha^{\mathrm{H}}\right)$ globins and $\beta^{\mathrm{S}}$ globins on a homozygous mouse $\beta^{\text {major }}$ deletional background $\left[\beta^{\mathrm{MDD}}\right]$ and symbolized as $\alpha^{\mathrm{H}} \beta^{\mathrm{S}}\left[\beta^{\mathrm{MDD}}\right]$ were kindly provided by M.E. Fabry (Albert Einstein College of Medicine). In these mice, $\beta^{S}$ globin levels are approximately $75 \%$ of all $\beta$ globin (29). We shall refer to these as $\beta^{\mathrm{S}}$ mice. The transgenic mice carrying the mutant gene were backcrossed for a total of eight generations with C57BL/6J mice (29). Systemic hematocrit (\%) in $\beta^{\mathrm{s}}$ mice is similar to that found for C57BL control mice ( $\beta^{\mathrm{S}}$ mice, $47.5 \pm 3$; controls, $46.5 \pm 2.1)(30)$.

Intravital microscopy. Male C57BL/6J control $(n=28)$ and transgenic $\beta^{S}(n=36)$ mice weighing approximately 25-30 g (4-6-months old), were used. The mice were maintained on a standard diet and water ad libitum. Mice were anesthetized intraperitoneally with $10 \%$ urethane and $2 \% \alpha$-chloralose in saline $(5 \mathrm{~mL} / \mathrm{kg})$. The animals were tracheostomized. The right jugular vein was cannulated for infusion of mAb's. In vivo microcirculatory observations were made in the open cremaster muscle preparation, prepared according to the method of Baez (31). The suffusion and maintenance of the mouse cremaster preparation was done as described previously (32). Briefly, the preparation was suffused with a bicarbonate Ringer's solution $(135.0 \mathrm{mMol} / \mathrm{L} \mathrm{NaCl}, 5.0$ $\mathrm{mMol} / \mathrm{L} \mathrm{KCl}, 27.0 \mathrm{mMol} / \mathrm{L} \mathrm{NaHCO}_{3}, 0.64 \mathrm{mMol} / \mathrm{L}$ $\mathrm{MgCl}_{2}$, and $11.6 \mathrm{mMol} / \mathrm{L}$ glucose); $\mathrm{pH}$ of the solution was adjusted to $7.35-7.4$ by continuous bubbling with $94.6 \% \mathrm{~N}_{2}$ and $5.6 \% \mathrm{CO}_{2}$. The osmolarity of the solution, as measured by a Microosmette (Precision Systems, Sudsbury, Massachusetts, USA) was $330 \mathrm{mOsm}$, as described for the mouse plasma (33). The temperature of the suffusion solution (flow rate, $5-6 \mathrm{~mL} / \mathrm{min}$ ) was maintained at $34.5-35^{\circ} \mathrm{C}$, and monitored by a telethermometer (YSI Inc., Yellowsprings, Ohio, USA). Microscopic observations were carried out using a Nikon microscope (model E400; Nikon Inc., Melville, New York, USA) equipped with a Dage-MTI CCD television camera (model CCD-300T-RC; Dage-MTI Inc., Michigan City, Indiana, USA) and a Sony U-matic video recorder (model VO5800; Sony, Teaneck, New Jersey, USA).

Intravital measurements were initiated immediately after the surgical procedure to exteriorize the tissue ( $\sim 15$ minutes). Red cell velocity $\left(\mathrm{V}_{\mathrm{rbc}}\right)$ and leukocyte flow dynamics were determined in postcapillary venules (diameter, $25-35 \mu \mathrm{m}$ ). Vessel luminal diameter (D) was measured online using an image shearing device (model 907; Instruments for Physiology and Medicine, San Diego, California, USA). $V_{\mathrm{rbc}}$ was measured along the vessel centerline using the "dual-slit" photodiode and a velocity cross-correlator $(34,35)$ (model $102 \mathrm{BF}$; Instruments for Physiology and Medicine). The centerline $V_{\mathrm{rbc}}$ was converted to the mean $V_{\mathrm{rbc}}$ across the vessel diameter using a conversion factor of $1.6\left(\mathrm{~V}_{\mathrm{rbc}} / \mathrm{V}_{\text {mean }}=1.6\right)$, originally described by Baker and Wayland (36), and later validated by Seki and Lipowsky (37). Volumetric flow rates (Q) were determined from $\mathrm{V}_{\text {mean }}$ and the vessel cross-sectional area $\left(\mathrm{BD}^{2} / 4\right)$ as described elsewhere $(36,38)$. Shear rates along the wall of microvessel of a given luminal diameter (D) were calculated using the relationship $=8 \mathrm{~V}_{\text {mean }} / \mathrm{D}(38)$.

Rolling leukocytes were defined as those leukocytes that distinctly roll along the endothelial surface. The rolling leukocytes can be easily distinguished because of their lower velocity compared with that of leukocytes and red cells in the flow (28). Rolling leukocyte velocity $(\mu \mathrm{m} / \mathrm{s})$ represented the time required for a rolling leukocyte to traverse a given length of venule. An average of rolling velocities of approximately ten leukocytes per venule was determined by frame-by-frame analysis of video replay. Rolling leukocyte flux (cells per minute) was determined as the number of leukocytes rolling through a given point in a vessel. A leukocyte was considered adherent if it remained stationary for longer than 30 seconds. Adherent leukocytes were counted along the length of a given venule and expressed as average number of cells per $100-\mu \mathrm{m}$ length 
of the vessel. Emigrated leukocytes were determined as the number of interstitial leukocytes in the field of view adjacent (within $\sim 30 \mu \mathrm{m}$ ) to venules.

Peripheral leukocyte counts on blood samples obtained from mice were determined using a Neubauer chamber as described previously (39).

Hypoxia/reoxygenation. Wild-type controls and $\beta^{\text {s mice }}$ were subjected to 2 - or 3-hour hypoxia using $10 \%$ oxygen, $0.5 \% \mathrm{CO}_{2}$, and balance nitrogen. After hypoxic periods, mice were returned to room air (reoxygenation). The protocol consisted of the following experimental groups. Group 1: This group ( $n=4$ each) served as normoxic controls (room air). Group 2: The mice $(n=4$ each) were subjected to 2 hours of hypoxia followed by 1 hour of reoxygenation. Group 3: The mice ( $n=4$ controls, $3 \beta^{\mathrm{S}}$ mice) were subjected to 2 hours of hypoxia and 2 hours of reoxygenation. Group 4: The mice $(n=3$ controls, $4 \beta^{\mathrm{S}}$ mice) were subjected to 3 hours of hypoxia. Group 5: The mice ( $n=3$ each) were subjected to 3 hours of hypoxia and 4 hours of reoxygenation. Group 6: Mice ( $n=4$ each) were subjected to 3 hours of hypoxia and 18 hours of reoxygenation. In both control and $\beta^{s}$ mice, hypoxia induced a decrease in arterial hemoglobin oxygen $\left(\mathrm{HbO}_{2}\right)$ saturation (monitored using Ohmeda oximeter and oxytip probe; model 3770; Ohmeda Inc., Madison, Wisconsin, USA) from the baseline line values of approximately $90 \%$ and $85 \%$, respectively, to less than $70 \%$ in each case. Reoxygenation resulted in a complete recovery of the arterial $\% \mathrm{HbO}_{2}$.

In separate experiments wherein the $\beta^{\mathrm{s}}$ mice were subjected to 3 hours of hypoxia, murine mAb's to Pselectin (RB40.34; PharMingen, San Diego, California, USA) or E-selectin (10E9.6; PharMingen) (each 100 $\mu \mathrm{g} /$ mouse) was infused via the jugular vein just before reoxygenation. Intravital observations were performed after 4 hours of reoxygenation.
Fluorochrome marker of oxidant generation. In selected experiments, we used oxidant-sensitive fluorochrome probe dihydrorhodamine 123 (DHR) (Molecular Probes Inc., Eugene, Oregon, USA) in the suffusate bathing the cremaster preparation. Stock solution of DHR in DMSO was stored at $-20^{\circ} \mathrm{C}$. Working solution of DHR $(10 \mu \mathrm{mol} / \mathrm{L})$ was made in bicarbonate Ringer (continuously bubbled with $94.6 \% \mathrm{~N}_{2}$ and $5.6 \% \mathrm{CO}_{2}$ ). The cremaster preparation was suffused with DHR for 15 minutes. DHR has been previously used to detect intracellular generation of $\mathrm{H}_{2} \mathrm{O}_{2}$ in a variety of cell types including vascular endothelium (40-42). In the presence of oxidants, nonfluorescent DHR is oxidized to fluorescent rhodamine 123 (RH123) that is localized in mitochondria. Oxidation of DHR is not due to $\mathrm{H}_{2} \mathrm{O}_{2}$ alone but may involve potential intracellular reactions involving secondary $\mathrm{H}_{2} \mathrm{O}_{2}$-dependent reactions (40). Fluorescent images were videotaped using Nikon microscope equipped with epifluorescence (model E400; Nikon Inc.) and a low-light sensitive Dage-MTI CCD-300 cooled TV camera in a fixed-gain mode. Images were digitized into a Macintosh Quadra 840AV computer (Apple Computer Inc., Cupertino, California, USA) with a Quickcapture card with auto gain off. NIHImage (Bethesda, Maryland, USA) was used to quantitate fluorescence intensities. To discern the spatial dimension of DHR fluorescence in venules, the profile of fluorescence intensity across vessel segments was examined (Figure 1). To determine differences in the fluorescence intensity in vessel endothelium, the average intensity in a $7 \times 7$ pixel area (gray level scale: $0-255$ ) was measured in the surrounding tissue and vessel lumen (background) and in endothelial cells of the vessel walls $(7-\mu \mathrm{m}$ width and $\sim 100-\mu \mathrm{m}$ length) of a given venular segment, as described elsewhere (43). The averaged values for each (background and vessel wall) were
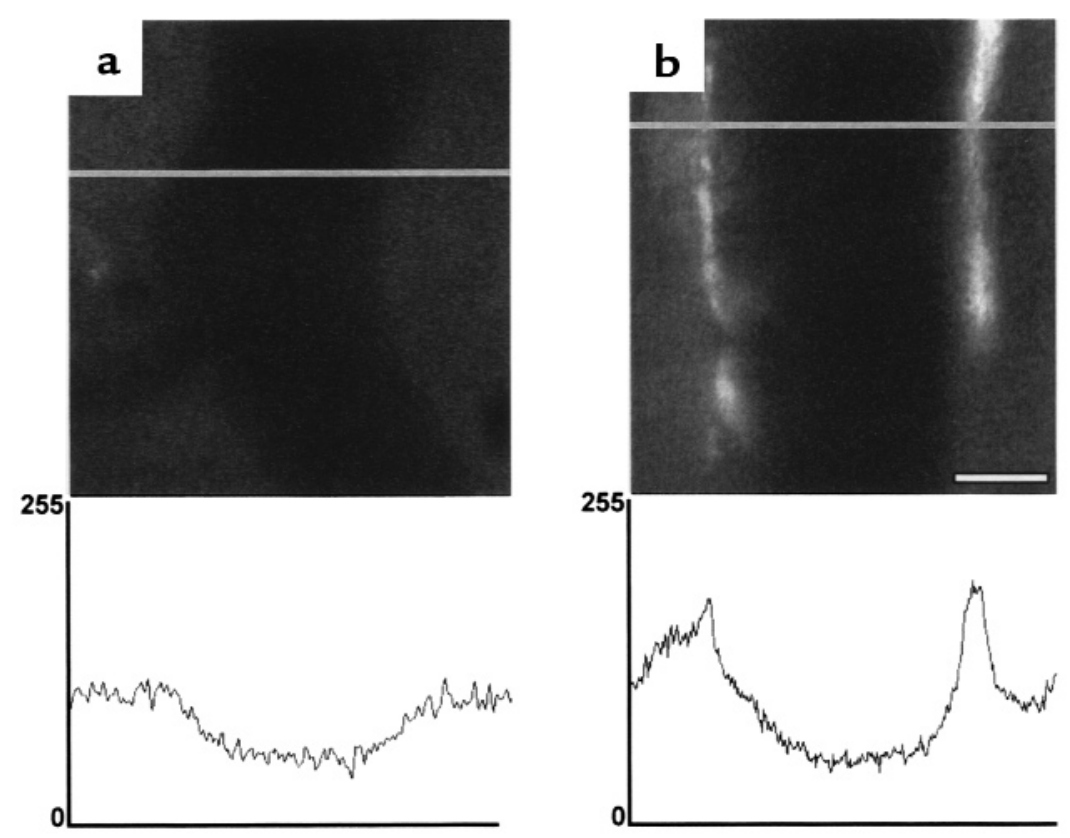
Figure 1
Representative fluorographs of cremas- ter venules in $\beta^{\text {s }}$ mice (top) and their corresponding DHR fluorescence pro- file analysis (bottom). (a) Normoxic control. (b) After 3 hours of hypoxia and 30 minutes of reoxygenation, the peaks of DHR activity correspond to fluorescence intensity in the vascular endothelial cells. Bar $=10 \mu \mathrm{m}$.




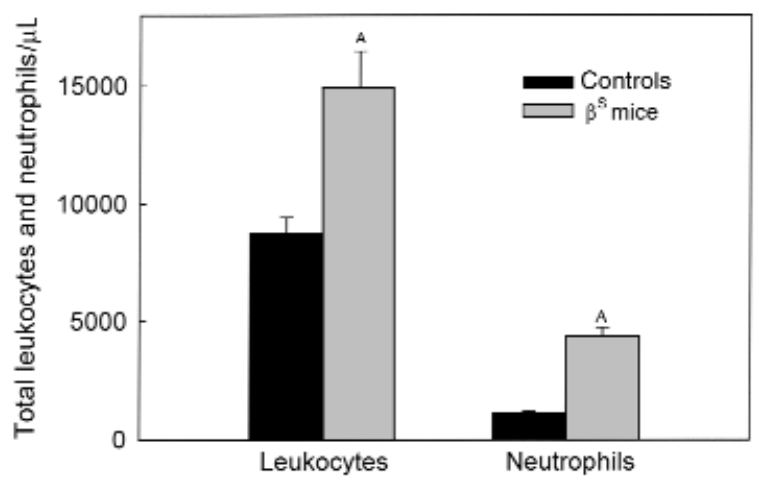

Figure 2

Peripheral leukocyte and neutrophil counts in control and $\beta^{\mathrm{s}}$ mice. AP $<0.005-0.003$

determined, and their difference (delta intensity, $\Delta \mathrm{I}$ ) was used to estimate the relative levels of oxidized DHR, indicative of $\mathrm{H}_{2} \mathrm{O}_{2}$ formation. Fluorescence intensities were measured in eight to 15 venular segments (total = 70 venular segments) under a given experimental condition for control and $\beta^{s}$ mice $(n=2$ each), i.e., normoxia, 3 hours of hypoxia plus 30 minutes of reoxygenation ( 15 minutes of cremaster exteriorization plus 15 minutes of DHR application), and 3 hours of hypoxia plus 4 hours of reoxygenation. In transgenic mice $(n=2)$ in which RH123 was used $(10 \mu \mathrm{mol} / \mathrm{L})$ as control, no significant differences in fluorescence intensity were evident after 3 hours of hypoxia plus 4 hours of reoxygenation compared with normoxic condition.

Statistical analysis. A total of 313 venules (in addition to fluorescence studies) were analyzed for various microcirculatory flow parameters. The number of venules in experimental groups ranged from 17 to 28 . Statistical analysis of the data was performed using one-way ANOVA, followed by Newman-Keuls multiple comparisons. Comparisons between groups (control versus transgenic) were made using the Student's $t$ test. Where tests for normality failed, or Bartlett's test for homogeneity of variance showed significant difference in the SD, nonparametric tests such as Kruskal-Wallis test for ANOVA or the Wilcoxon two-sample test were used. $P<$ 0.05 was considered significant. The statistical analysis was performed using Statgraphics Plus 3.0 program for Windows (Manugistics Inc., Rockville, Maryland, USA).

\section{Results}

Peripheral leukocyte and neutrophil counts. To explore whether transgenic sickle mice are predisposed to a proinflammatory state, we compared peripheral leukocyte and neutrophil counts in $\beta^{\mathrm{s}}$ mice and control wild-type (C57) mice. During steady-state conditions, the transgenic mice showed a 1.7-fold increase in the peripheral leukocyte counts $(P<0.005)$ and an almost threefold increase in neutrophil counts $(P<$ 0.003 ) compared with control mice (Figure 2$)$. The neutrophil counts in control mice are in agreement with previous studies $(44,45)$.
Hemodynamic parameters and leukocyte flow dynamics in transgenic sickle mice under steady state conditions. Hemodynamic parameters $\left(\mathrm{V}_{\mathrm{rbc}}\right.$, wall shear rates, and $\left.\mathrm{Q}\right)$ were examined in the postcapillary venules (diameter, 20-40 $\mu \mathrm{m})$ in the cremaster muscle preparations of wild-type controls and $\beta^{S}$ mice (Table 1). Under steady-state (normoxic) conditions, the $\beta^{\mathrm{S}}$ mice showed $50 \%$ lower $\mathrm{V}_{\mathrm{rbc}}$ and wall shear rates (each $P<0.0001$ ) compared with control wild-type mice, accompanied by a greater than $50 \%$ decrease $(P<0.001)$ in the venular blood flow (Table $1)$. Of interest is the observation that in the $\beta^{s}$ mice, the higher leukocyte counts were associated with approximately a $40 \%$ decrease in leukocyte rolling velocity $(P<$ $0.0001)$ and more than a twofold increase in the baseline leukocyte rolling flux $(P<0.0001)$ compared with controls (Figures 3 and $4 \mathrm{a}$ ). The increase in leukocyte rolling flux in normoxic $\beta^{\mathrm{S}}$ mice was associated with increased number of adherent leukocytes $(P<0.01)$ (Figure 4b).

The effect of hypoxia-reoxygenation. Because hypoxia is expected to cause increased in vivo sickling and ischemia only in the transgenic sickle mice, we evaluated whether hypoxia followed by reoxygenation would trigger a greater response in the $\beta^{\mathrm{s}}$ sickle mice. Mice were subjected to 2 or 3 hours of hypoxia using $10 \%$ oxygen in a breathing mixture (plus $0.5 \% \mathrm{CO}_{2}$ and balance nitrogen) followed by return to ambient (normoxic) conditions.

The effect of hypoxia. Hemodynamic parameters and leukocyte flow dynamics were examined in control and $\beta^{\mathrm{s}}$ mice subjected to 3 hours of hypoxia. Hypoxia alone did not cause significant changes in $V_{r b c}$, wall shear rates, or the flow compared with the baseline values in either group of mice (Table 1). Hypoxia resulted in a slight but insignificant increase in the leukocyte rolling velocity in both controls and $\beta^{\mathrm{S}}$ mice (Figure 3). Compared with the baseline normoxic values, leukocyte rolling flux showed an increase in controls $(P<0.05)$ but not in the $\beta^{\text {s }}$ mice (Figure 4a). There was a decrease in adherent leukocytes that was significant $(P<0.05)$ for $\beta^{\mathrm{s}}$ mice (Figure $\left.4 \mathrm{~b}\right)$.

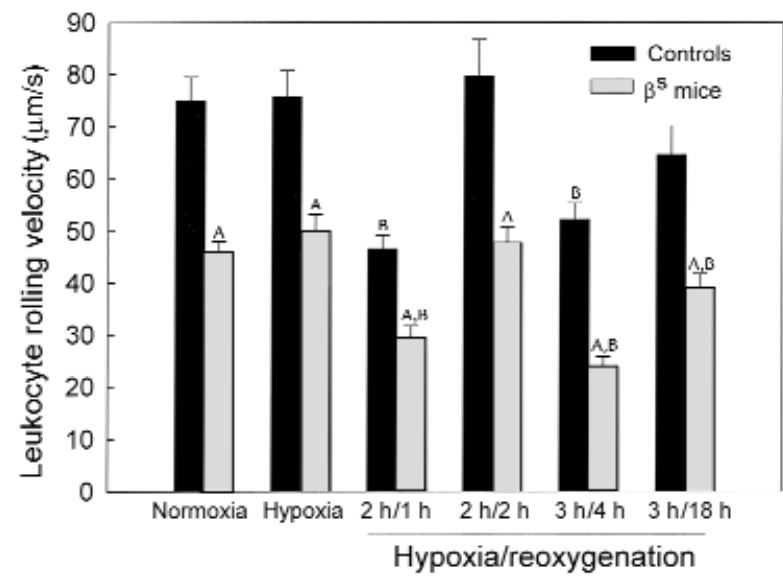

Figure 3

The effect of hypoxia/reoxygenation on leukocyte rolling velocity in control and $\beta^{S}$ mice. ${ }^{A} P<0.001$ versus corresponding wild-type controls (Student's $t$ test); ${ }^{B} P<0.05$ versus respective normoxic values (Newman-Keuls multiple comparisons). 
Table 1

The effect of hypoxia/reoxygenation on hemodynamic parameters in the microcirculation of wild-type controls and $\beta^{\mathrm{s}}$ mice

\begin{tabular}{|c|c|c|c|c|c|c|}
\hline \multirow[t]{2}{*}{ Parameter } & \multirow[t]{2}{*}{ Normoxia } & \multirow[t]{2}{*}{ Hypoxia } & \multicolumn{4}{|c|}{ Hypoxia/reoxygenation } \\
\hline & & & 2 hours $/ 1$ hour & 2 hours/ 2 hours & 3 hours $/ 4$ hours & 3 hours $/ 18$ hours \\
\hline \multicolumn{7}{|c|}{ Venular diameter, $\mu \mathrm{m}$} \\
\hline Wild-type & $30.1 \pm 1.7(24)^{\mathrm{A}}$ & $31.8 \pm 1.2(21)$ & $26.9 \pm 1.3(26)$ & $27.4 \pm 1.4(24)$ & $28.6 \pm 1.5(17)$ & $28.9 \pm 1.0(20)$ \\
\hline Transgenic & $28.7 \pm 1.1(28)$ & $32.8 \pm 1.3(23)$ & $27.1 \pm 1.2(20)$ & $26.6 \pm 0.9(28)$ & $27.1 \pm 0.8(19)$ & $27.3 \pm 1.1(23)$ \\
\hline \multicolumn{7}{|c|}{ Red cell velocity, mm/s } \\
\hline Wild-type & $4.0 \pm 0.35$ & $4.6 \pm 0.46$ & $1.7 \pm 0.14^{B}$ & $2.6 \pm 0.39^{B}$ & $2.6 \pm 0.32$ & $3.6 \pm 0.46$ \\
\hline Transgenic & $1.9 \pm 0.15^{C}$ & $2.1 \pm 0.19^{C}$ & $1.2 \pm 0.09^{\mathrm{B}, \mathrm{C}}$ & $1.5 \pm 0.08^{\mathrm{B}, \mathrm{C}}$ & $1.4 \pm 0.16^{\mathrm{B}, \mathrm{C}}$ & $1.9 \pm 0.2^{C}$ \\
\hline \multicolumn{7}{|c|}{ Wall shear rate, $\mathrm{s}^{-1}$} \\
\hline Wild-type & $684 \pm 64$ & $737 \pm 77$ & $311 \pm 26^{B}$ & $485 \pm 79^{B}$ & $397 \pm 30^{B}$ & $598 \pm 71$ \\
\hline Transgenic & $339 \pm 30^{c}$ & $323 \pm 26^{C}$ & $235 \pm 21^{B, C}$ & $282 \pm 18$ & $247 \pm 26^{B, C}$ & $364 \pm 46^{C}$ \\
\hline \multicolumn{7}{|c|}{ Venular blood flow, $\mathrm{nL} / \mathrm{s}$} \\
\hline Wild-type & $1.96 \pm 0.31$ & $2.38 \pm 0.31$ & $0.66 \pm 0.09^{\mathrm{B}}$ & $1.0 \pm 0.15^{\mathrm{B}}$ & $0.96 \pm 0.18^{B}$ & $1.65 \pm 0.29$ \\
\hline Transgenic & $0.78 \pm 0.08^{c}$ & $1.21 \pm 0.15^{C}$ & $0.47 \pm 0.05^{\mathrm{B}}$ & $0.54 \pm 0.05^{\mathrm{B}, \mathrm{C}}$ & $0.5 \pm 0.08^{B, C}$ & $0.77 \pm 0.15^{C}$ \\
\hline
\end{tabular}

Values are mean \pm SE. ANumbers in parentheses represent the number of venules examined for hemodynamic parameters. ${ }^{\mathrm{B}} P<0.05$ versus respective normoxic values. ${ }^{C} P<0.04-0.0001$ versus corresponding wild-type controls.

\section{The effect of reoxygenation}

Hemodynamic parameters. Table 1 depicts hemodynamic parameters after hypoxia/reoxygenation in wild-type controls and $\beta^{s}$ mice. Two hours of hypoxia followed by 1 hour of reoxygenation induced significant decreases in $\mathrm{V}_{\mathrm{rbc}}$, wall shear rates, and $\mathrm{Q}(P<0.05)$ in both groups of mice (Table 1). After 2 hours of reoxygenation, these parameters showed a significant improvement in control mice $(P<0.05)$, but not in the $\beta^{\text {s }}$ mice. When 3 hours of hypoxia was followed by 4 hours of reoxygenation, $V_{\mathrm{rbc}}$, wall shear rates and the flow in the $\beta^{\mathrm{S}}$ mice showed a significantly greater decline $(\sim 40 \%)$ compared with controls $(P<0.03-0.001)$. After 18 hours of reoxygenation, $V_{\mathrm{rbc}}$, wall shear rates, and $\mathrm{Q}$ in $\beta^{\mathrm{s}}$ mice remained lower compared with those of corresponding controls $(P<0.01-0.003)$ but were not significantly different from the normoxic baseline values (Table 1).

Leukocyte rolling velocity, rolling fluxes, and adhesion. As is evident from Figure 3 , in both control and $\beta^{\text {s }}$ mice, leukocyte rolling velocity in normoxic conditions and after hypoxia-reoxygenation essentially followed the same pattern as shown for wall shear rates in Table 1. Apparently, at least within the range depicted in Table 1 , wall shear rates seem to affect leukocyte rolling velocity in both groups.

Two hours of hypoxia followed by 1 hour of reoxygenation induced significant increases in the rolling fluxes in both groups of mice (Figure 4a). However, the $\beta^{\mathrm{S}}$ mice had an approximately $50 \%$ greater leukocyte rolling flux $(P<0.001)$, accompanied by more than 2.5 fold greater numbers of adherent leukocytes $(P<0.001$; Figure $4 \mathrm{~b}$ ) compared with controls. After 2 hours of reoxygenation, the rolling fluxes, as well as the number of adherent leukocytes, decreased in both groups. When 3 hours of hypoxia was followed by 4 hours of reoxygenation, the $\beta^{S}$ mice showed a decrease in the rolling flux compared with the baseline normoxic val- ues $(P<0.05)$. Notably, at 4 hours of reoxygenation, the decrease in the rolling flux in the $\beta^{\mathrm{S}}$ mice was accompanied by a more than twofold increase in adherent leukocytes over the baseline (normoxic) values for these mice $(P<0.05)$ and a 2.6-fold increase over the values for controls subjected to the same protocol $(P<0.0001$; Figure 4b). Further, at 18 hours of reoxygenation, the $\beta^{\mathrm{s}}$ mice showed a 1.6-fold increase in adherent leukocytes compared with the baseline normoxic values $(P<0.05)$, and almost threefold greater adhesion $(P<0.0001)$ compared with corresponding values for control mice (Figure 4b), confirming an adverse effect of reoxygenation in the transgenic mice. Thus, in the $\beta^{\mathrm{S}}$ mice, a greater proportion of rolling leukocytes was able to adhere to the endothelium with increases in hypoxic and reoxygenation periods. On the other hand, at 18 hours of reoxygenation, in control mice, both the leukocyte flux and adhesion showed significant decreases $(P<0.05)$ compared with the values at 4 hours of reoxygenation and were closer to the baseline (normoxic) values.

Leukocyte emigration. In transgenic sickle mice, inflammatory response to hypoxia/reoxygenation was evident by leukocyte extravasation (Figure 5). In contrast, wildtype controls showed no significant changes in leukocyte emigration with reoxygenation. Transgenic mice showed an approximately fourfold increase in emigrated leukocytes after 4 hours of reoxygenation $(P<0.01$ versus normoxic values and corresponding wild-type controls) and a greater than 15-fold increase after 18 hours of reoxygenation $(P<0.0001$ versus normoxic values and corresponding wild-type controls) (Figure 6).

The effect of anti-P-and anti-E-selectin antibodies. Next, we investigated whether hypoxia/reoxygenation-induced increase in leukocyte recruitment in $\beta^{\mathrm{s}}$ mice was preventable using mouse-specific anti-P- or anti-E-selectin antibodies. mAb's to murine P-selectin (RB40.34) or Eselectin (10E9.6) (each $100 \mu \mathrm{g} /$ mouse) was infused just 


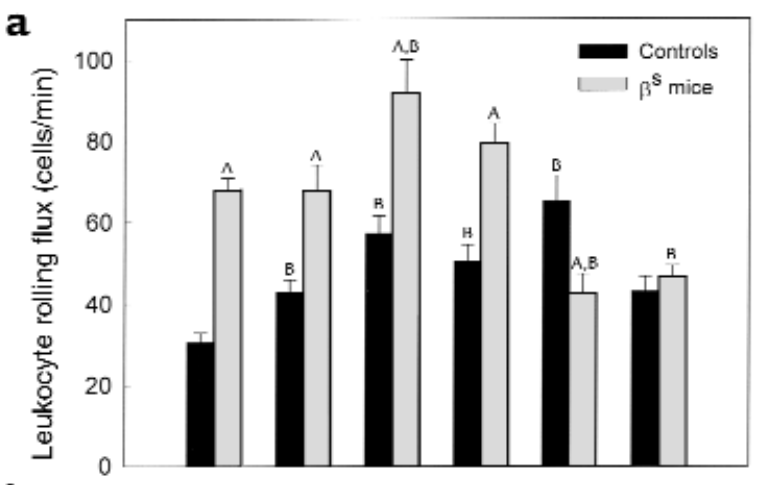

b

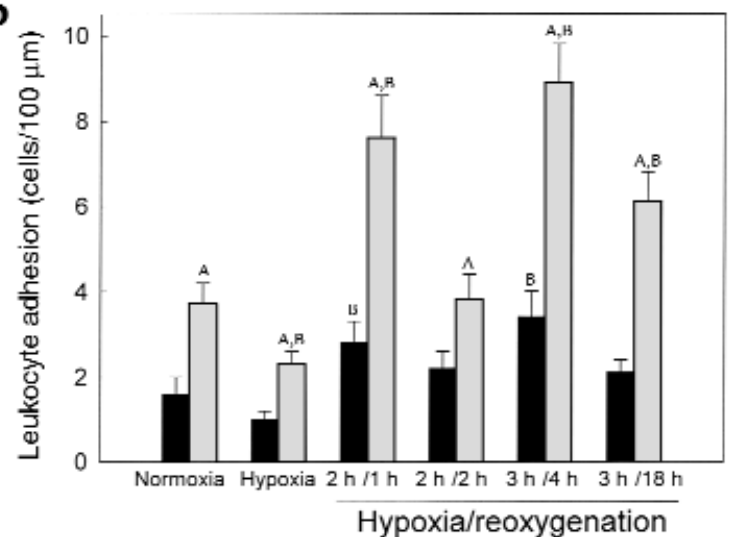

Figure 4

(a) The effect of hypoxia/reoxygenation on leukocyte rolling flux in control and $\beta^{s}$ mice. (b) The effect of hypoxia/reoxygenation on leukocyte adhesion in control and $\beta^{S}$ mice. ${ }^{A} P<0.01-0.0001$ versus corresponding wild-type controls (Wilcoxon two-sample test); ${ }^{B} P<0.05$ versus respective normoxic values (Kruskal-Wallis test for ANOVA).

before reoxygenation. Intravital observations were performed after 4 hours of reoxygenation.

Wall shear rates showed approximately a twofold increase in anti-P-selectin-treated $\beta^{S}$ mice compared with untreated mice subjected to hypoxia/reoxygenation protocol (from $247 \pm 26 \mathrm{~s}^{-1}$ to $468 \pm 72 \mathrm{~s}^{-1} ; P<$ $0.004)$, whereas anti-E-selectin had no effect on wall shear rates $\left(283 \pm 23 \mathrm{~s}^{-1} ; P>0.16\right.$ versus untreated group) (Table 2). Evidently, the increases in $\mathrm{V}_{\mathrm{rbc}}$ and wall shear rates in the anti-P-selectin group (Table 2) were consequent to complete inhibition of leukocyte rolling and adhesion as shown in Figure 7, a and b, and there was no leukocyte emigration into the interstitial tissue. In contrast, there was no effect of anti-E-selectin $\mathrm{mAb}$ on these variables compared with the untreated group (Figure 7, $\mathrm{a}$ and $\mathrm{b}$ ).

Endothelial oxidant production. In selected experiments, DHR fluorescence intensity differences $(\Delta \mathrm{I})$ were determined between the background and the venular endothelium (Figure 1) during normoxia, at 3 hours hypoxia plus 30 minutes of reoxygenation and after 3 hours hypoxia plus 4 hours of reoxygenation. Figure 8 shows that maximal differences in the averaged DHR fluorescence intensity were recorded in $\beta^{\text {s }}$ mice after 3 hours of hypoxia plus 30 minutes of reoxygenation $(P<0.0002$ versus respective controls). After 3 hours of hypoxia and 4 hours of reoxygenation, $\Delta \mathrm{I}$ for $\beta^{\mathrm{s}}$ mice showed a decrease, but was still significantly higher than the corresponding control values $(P<0.0001)$, as well as the normoxic values $(P<0.05)$. In contrast, $\Delta \mathrm{I}$ for controls at 3 hours hypoxia plus 4 hours reoxygenation was not significantly different from the control normoxic values. These results show a clear evidence of DHR oxidation in endothelial cells under hypoxia/reoxygenation and an exaggerated response to hypoxia and reoxygenation in the $\beta^{\mathrm{S}}$ mice. No DHR oxidation was evident in leukocytes.

\section{Discussion}

Previous studies in normal animals have shown that the inflammatory response evoked by ischemia/reperfusion is characterized by increased rolling flux and adhesion of leukocytes in postcapillary venules followed by their extravasation (3). In the present studies, we have compared the effect of hypoxia/reoxygenation in control and transgenic sickle mice to test our hypothesis that hypoxia-induced in vivo red cell sickling followed by reoxygenation would result in an exaggerated inflammatory response in the transgenic mice,
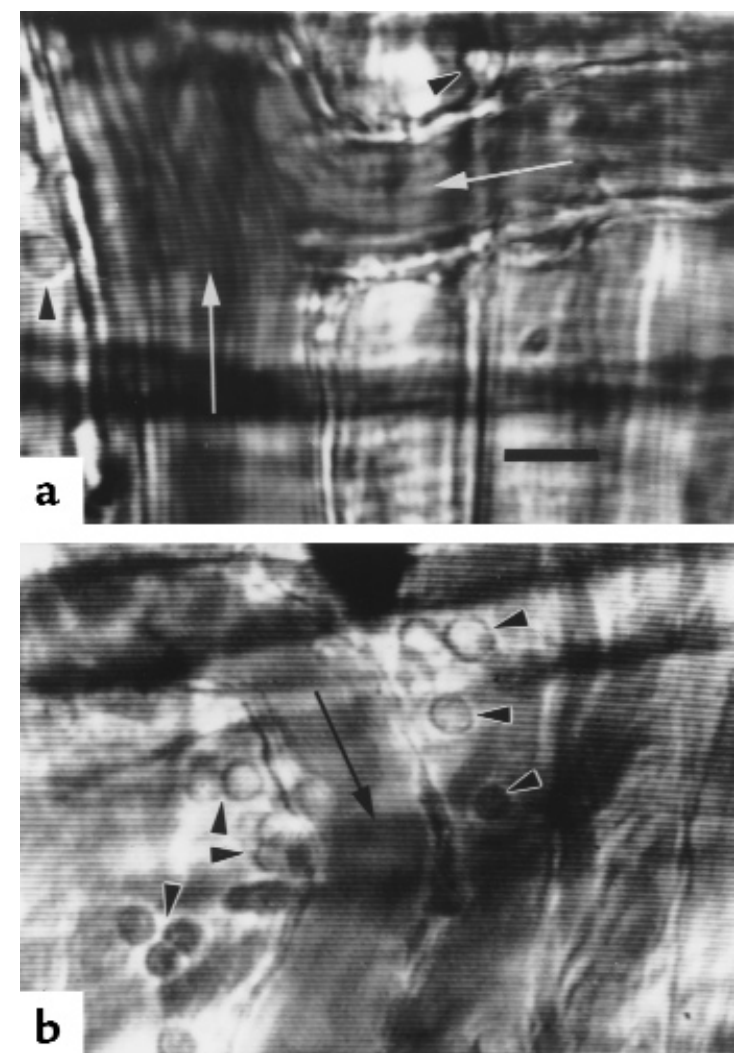

Figure 5

Photomicrographs of cremaster venules after 3-hour hypoxia plus 18 hours of reoxygenation. (a) Normal mouse shows few emigrated leukocytes (arrowheads) in the cremaster. (b) In contrast, $\beta^{\mathrm{s}}$ mouse shows a large number of emigrated leukocytes adjacent to a venule (arrowheads). Large arrows depict the flow direction. Next to the venule in the upper middle part is the image of photodiode fibers for $V_{\text {rbc }}$ measurement. Bar $=20 \mu \mathrm{m}$. 


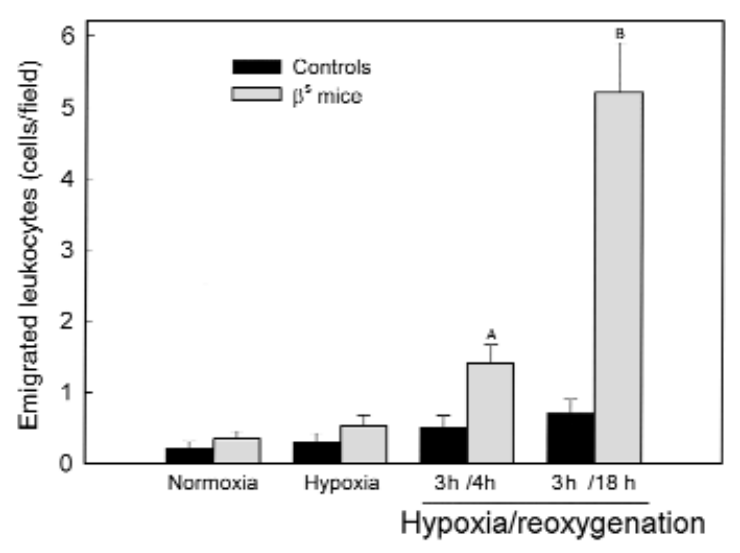

Figure 6

The effect of hypoxia/reoxygenation on leukocyte emigration in control and $\beta^{S}$ mice. ${ }^{A} P<0.01$ and ${ }^{B} P<0.0001$ versus corresponding wild-type controls (Wilcoxon two-sample test).

but not in control mice. We did this by inducing modest hypoxia with exposure to $10 \%$ oxygen. Thus, our approach markedly differs from previous studies in which reperfusion injury was induced after acute ischemia (i.e., complete or significant occlusion of blood supply). In our model, however, hypoxia/reoxygenation is not expected to result in much of an inflammatory response in normal mice in which no sickling (and therefore no ischemia) is expected. This contrast is well established by our results that demonstrate, for the first time to our knowledge, that compared with control mice, hypoxia/reoxygenation induces a exaggerated inflammatory response in transgenic sickle mice.

In the $\beta^{\text {S }}$ mice, the higher peripheral leukocyte counts and a greater baseline leukocyte flux are indicative of endothelial activation and a proinflammatory state even under ambient air conditions that also characterizes human sickle cell disease (10-12). The baseline $\mathrm{V}_{\mathrm{rbc}}$, wall shear rates, and the volumetric flow in the $\beta^{\mathrm{S}}$ mice were significantly lower than those in control mice, further suggesting that increased leukocyte counts and the rolling fluxes along with red cell rheological abnormalities contribute significantly to the impaired microvascular flow in the transgenic mice. When 2 hours of hypoxia was followed by 1 hour of reoxygenation, there was a significant increase in the leukocyte rolling fluxes in both control and $\beta^{\mathrm{s}}$ mice. Although the $\beta^{S}$ mice showed a greater leukocyte rolling flux and adhesion compared with controls (see Figure 4, a and b), notably there was no leukocyte emigration (data not shown) after 2 hours of reoxygenation in either group. A greater number of adherent leukocytes and a distinct inflammatory response were observed in the $\beta^{\mathrm{S}}$ mice when 3 hours of hypoxia was followed by 4 hours of reoxygenation. In the $\beta^{\text {s mice, }}$ the inflammatory response was characterized by leukocyte emigration that increased significantly with increased duration of reoxygenation period to 18 hours (see Figure 5). Control mice showed no increase in extravasated leukocytes over the baseline values at any period of reoxygenation, confirming a lack of major inflammatory response.

The greater leukocyte rolling fluxes, adhesion, and emigration in the $\beta^{\mathrm{S}}$ mice after reoxygenation are likely due to relative ischemia resulting from abnormal red cell rheology (intravascular sickling) during the hypoxic period. Therefore, the relative unresponsiveness of normal mice to exposure of equivalent hypoxia strongly suggests that red cell sickling is etiologic here.

The increased abnormality with reoxygenation after hypoxia is consistent with the known understanding of reperfusion. Recent studies by Osarogiagbon et al. (46) have shown that both lipid peroxidation and $\mathrm{OH}$ radical generation do occur in the same sickle mouse model under the same experimental conditions. In the present studies, we have monitored generation of oxidants in cremaster venules using DHR, a $\mathrm{H}_{2} \mathrm{O}_{2}$ sensitive fluorochrome. As reported previously, generation of $\mathrm{H}_{2} \mathrm{O}_{2}$ in vascular endothelial cells after application of inflammatory stimuli results in oxidation of nonfluorescent DHR to fluorescent rhodamine (40). The results show maximal DHR oxidation in vascular endothelial cells of $\beta^{s}$ mice after a brief reoxygenation following hypoxia. The

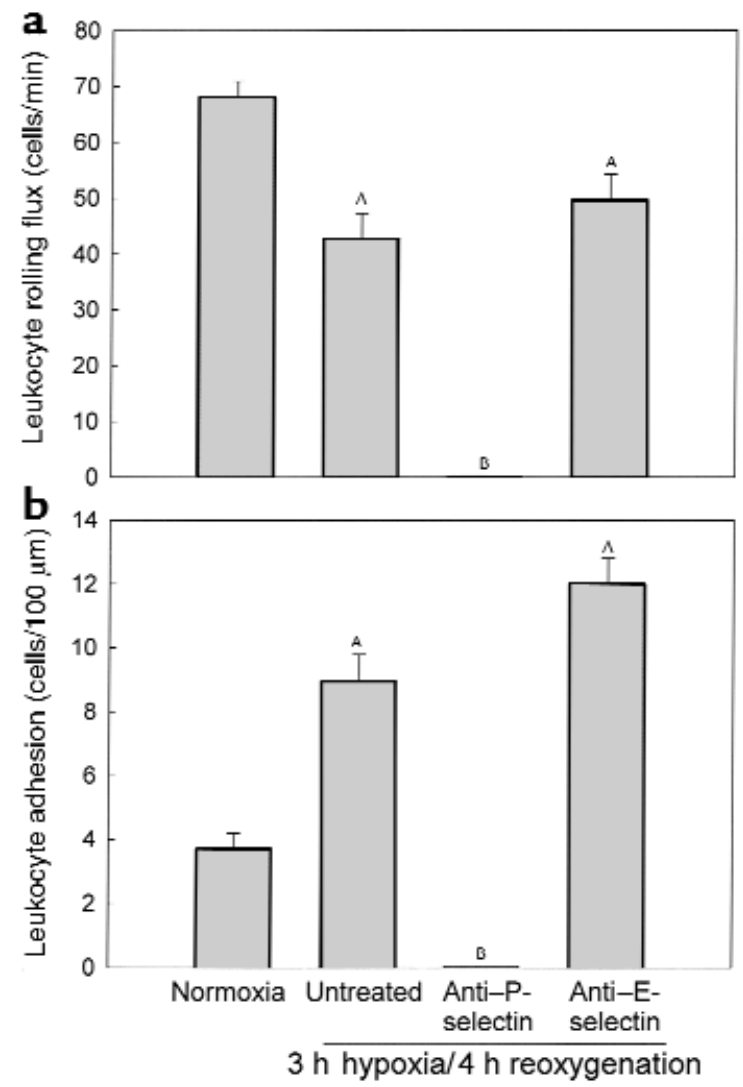

Figure 7

(a) Hypoxia/reoxygenation in the $\beta^{\mathrm{s}}$ mice: the effect of anti-P- and anti-E-selectin antibodies on leukocyte rolling flux. (b) Hypoxia/reoxygenation in the $\beta^{\mathrm{S}}$ mice: the effect of anti-P- and anti-E-selectin antibodies on leukocyte adhesion. ${ }^{A} P<0.05$ versus normoxic values; ${ }^{\mathrm{B}} P<0.05$ versus other groups (Newman-Keuls multiple comparisons). 
Table 2

The effect of anti-P- and anti-E-selectin antibodies on $\mathrm{V}_{\mathrm{rbc}}$ and wall shear rates in $\beta^{\mathrm{s}}$ mice

\begin{tabular}{lccc}
\hline Mice & No. of venules & $\begin{array}{c}\mathrm{V}_{\text {rbc }} \\
\mathrm{mm} / \mathrm{s}\end{array}$ & $\begin{array}{c}\text { Wall shear rates } \\
\mathrm{s}^{-1}\end{array}$ \\
$\begin{array}{l}\text { Wild-type } \\
\text { (normoxia }^{\mathrm{A}} \text { ) }\end{array}$ & 24 & $4.0 \pm 0.35$ & $684 \pm 64$ \\
$\beta^{\mathrm{S}}$ mice $^{\text {1. Normoxia }}{ }^{\mathrm{A}}$ & 28 & $1.9 \pm 0.15^{\mathrm{B}}$ & $339 \pm 30^{\mathrm{B}}$ \\
$\begin{array}{l}\text { 2. } 3 \text {-hour hypoxia/4 hour reoxygenation } \\
\text { i. Untreated }\end{array}$ & 19 & $1.4 \pm 0.16^{\mathrm{C}}$ & $247 \pm 26^{\mathrm{C}}$ \\
$\begin{array}{l}\text { ii. Anti-P-selectin } \\
\text { iii. Anti-E-selectin }\end{array}$ & 17 & $3.2 \pm 0.54^{\mathrm{D}}$ & $468 \pm 72^{\mathrm{D}}$ \\
& 23 & $1.4 \pm 0.1^{\mathrm{C}}$ & $283 \pm 23$
\end{tabular}

Values are mean \pm SE. ${ }^{A}$ Based on the values given in Table $1 .{ }^{B} P<0.001$ versus wild-type controls. ${ }^{C} P<0.05$ versus normoxic values in $\beta^{\mathrm{s}}$ mice. D $P<0.002-0.006$ versus untreated and $\mathrm{E}$-selectin groups.

reoxygenation period (30 minutes) is attributable to cremaster tissue exteriorization ( 15 minutes) under ambient conditions and the application of DHR suffusate (15 minutes) after the hypoxic period. Further, after 4 hours of reoxygenation, $\beta^{\mathrm{S}}$ mice show a distinct decrease in DHR oxidation, but an increase in adherent and emigrated leukocytes. This may indicate that initial oxidative stress subsequently results in endothelial expression of adhesion molecules. Also, DHR oxidation in $\beta^{\mathrm{S}}$ mice is distinctly exaggerated compared with normal mice, which may also account for the observed differences in leukocyte-endothelium interactions between the two groups. Thus, our observations provide evidence of DHR oxidation in endothelial cells under the described experimental conditions, with distinct differences between normal and $\beta^{\mathrm{S}}$ mice. However, DHR fluorescence results must be interpreted cautiously, as future studies will be needed to obtain direct evidence for $\mathrm{H}_{2} \mathrm{O}_{2}$ generation in vivo (e.g., decreased fluorescence after catalase infusion).

These results strongly suggest that the overall process is related to sickling as the proximate event. The specific factor that stimulates endothelium may be sickled cells or other activating effects that accompany the proximate sickling event, i.e., oxidants generated by leukocytes, cytokine release, and so forth. These are novel findings with potential pathophysiological significance that indicate that both red cell rheology and leukocyte-endothelium interaction will contribute to the pathology in transgenic sickle mice. Leukocyte recruitment in postcapillary venules is known to increase vascular resistance dramatically (47), and may affect recovery from ischemic episodes (48). Future studies will be needed to ascertain the time sequence of peroxide generation and the expression of endothelial adhesion molecules after hypoxia/reoxygenation.

Given that rolling precedes firm adhesion and recruitment of leukocytes, inhibition of initial leukocyte rolling may present a therapeutic opportunity to prevent leukocyte-dependent inflammation and damage in a particular tissue (49). The results of the present studies demonstrate that infusion of monoclonal murine P-selectin antibody before reoxygenation completely abolished leukocyte rolling, adhesion, and extravasation in transgenic sickle mice. In contrast, at least within the time frame of our experimental protocol, infusion of monoclonal murine E-selectin antibody affected neither leukocyte rolling flux nor reoxygenation-induced inflammatory response in the cremaster muscle of the $\beta^{\mathrm{S}}$ mice.

Our results are in overall agreement with previous studies depicting P-selectin as the dominant receptor for leukocyte rolling in postischemic venules $(4,25)$, although species-to-species and organ-to-organ variations cannot be ruled out $(50,51)$. Moreover, our results are supported by recent studies in gene-targeted mice. For example, in P-selectin-deficient mice, baseline leukocyte rolling was completely absent in mesenteric venules, and stimulation with calcium ionophore or with $\mathrm{H}_{2} \mathrm{O}_{2}$ failed to have any detectable effect (44). Kanwar et al. (51), using the cremaster muscle preparation, showed that in contrast to wild-type mice, reperfusion did not induce any increase in leukocyte recruitment in P-selectin-deficient mice. Also, a mouse-specific anti-P-selectin antibody abolished reperfusion-induced leukocyte accumulation in wildtype mice. On the other hand, E-selectin-deficient mice show normal baseline leukocyte rolling, before or after stimulation with TNF-" (52). Also, there is no evidence in the literature for a role of E-selectin in reperfusioninduced injury in the muscle microvasculature (51).

Because of endothelial injury and activation in transgenic sickle mice (53), it is plausible that low levels of P-selectin are expressed even during basal conditions, which may result in the greater baseline leukocyte rolling flux in these mice. Increased oxygen radical generation, as exemplified by peroxynitrite formation in the $\beta^{\mathrm{S}}$ mice (54), could potentially induce P-selectin

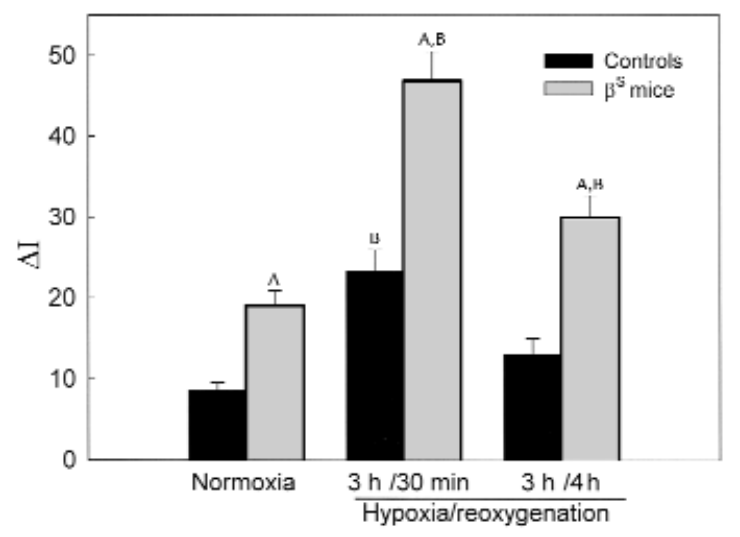

Figure 8

Differences in dihydrorhodamine 123 (DHR) intensity $(\Delta \mathrm{I})$ between background and venular endothelial cells in normal and $\beta^{\mathrm{s}}$ mice during normoxia and after hypoxia/reoxygenation. $\beta^{\mathrm{s}}$ mice showed greater $\Delta \mathrm{l}$ compared with corresponding wild-type controls in any experimental group ( $\left.{ }^{A} P<0.0002-0.0001\right)$, with maximal $\Delta$ l recorded at 30 minutes after the hypoxic period. ${ }^{\mathrm{B}} P<0.05$ compared with respective normoxic values (Newman-Keuls multiple comparisons). 
expression on the activated endothelium (3). Ley and coworkers $(55,56)$ showed that although baseline leukocyte rolling was totally absent in the cremaster muscle preparation of $\mathrm{P}$-selectin-deficient mice for first 30-60 minutes, significant leukocyte rolling was seen by 80 minutes. This delayed onset of leukocyte rolling was L-selectin dependent. Thus, we cannot rule out a role of L-selectin, as we have not examined timedependent leukocyte flow dynamics in the exteriorized cremaster tissue of $\beta^{\mathrm{S}}$ mice receiving P-selectin antibody before reoxygenation.

In the present studies, the choice of the transgenic $\beta^{\mathrm{s}}$ mouse that exhibits a slower progression of the disease compared with transgenic-knockout mice expressing exclusively human "- and $\beta^{\text {s }}$ globins (57) enabled us to investigate the effect of relatively long duration of hypoxia, and thereby establish the role of relative ischemia (sickling-induced)/reoxygenation in leukocyte-endothelium interactions. In contrast, greater pathology of the more severe knockout model may already be affected by leukocyte and endothelial activation owing to extensive intravascular sickling and recurring vasoocclusive events, and hypoxia may only worsen the pathology irretrievably. Thus, our studies show the practicality of using a transgenic sickle mouse with mild pathology in this type of hypoxia/reoxygenation studies.

In conclusion, our studies provide evidence for a proinflammatory state in the $\beta^{\mathrm{s}}$ mice, and demonstrate for the first time that hypoxia/reoxygenation, depending on the duration of hypoxia, can result in a distinct inflammatory response in the $\beta^{\mathrm{S}}$ mice. Consistent with this, the present studies show evidence of oxidant generation in endothelial cells, corroborating parallel biochemical evidence for oxidant stress related to reperfusion injury in $\beta^{\mathrm{S}}$ mice subjected to hypoxia/reoxygenation (46). Thus, in the $\beta^{s}$ mice, a chronic proinflammatory condition associated with red cell rheological abnormalities would result in endothelium activation. The implications of this are that the consequent facilitation of leukocyteendothelium interactions, and presumably red cellendothelium interaction via VCAM-1 (58), would contribute to vasoocclusive complications of this disease.

\section{Acknowledgments}

The authors express thanks to X. Liu for invaluable help in the analysis of leukocyte flow dynamics, and $\mathrm{M}$. Cammer (Analytical Imaging Facility) for assistance and advice in image analysis. This work was supported by NIH grant PO1 HL-55552.

1. Hebbel, R.P. 1991. Beyond hemoglobin polymerization: the red blood cell membrane and sickle disease pathophysiology. Blood. 77:214-237.

2. Kaul, D.K., Fabry, M.E., and Nagel, R.L. 1996. The pathophysiology of vascular obstruction in the sickle syndromes. Blood Rev. 10:29-44.

3. Grisham, M.B., Granger, D.N., and Lefer, D.J. 1998. Modulation of leukocyte-endothelial interactions by reactive metabolites of oxygen and nitrogen: relevance to ischemic heart disease. Free Radic. Biol. Med. 25:404-433.

4. Granger, D.N. 1999. Ischemia-reperfusion: mechanisms of microvascular dysfunction and the influence of risk factors for cardiovascular disease. Microcirculation. 6:167-178.

5. Klug, P.P., Kaye, N., and Jensen, W.N. 1982. Endothelial cell and vascular damage in the sickle cell disorders. Blood Cells. 8:175-184.
6. Sowemimo-Coker, S.O., Meiselman, H.J., and Francis, R.B., Jr. 1989. Increased circulating endothelial cells in sickle cell crisis. Am. J. Hematol. 31:263-265

7. Solovey, A., et al. 1997. Circulating activated endothelial cells in sickle cell anemia. N. Engl. J. Med. 337:1584-1590.

8. Conger, J.D., and Weil, J.V. 1995. Abnormal vascular function following ischemia-reperfusion injury. J. Investig. Med. 43:431-442.

9. Solovey, A., Gui, L., Key, N.S., and Hebbel, R.P. 1998. Tissue factor expression by endothelial cells in sickle cell anemia. J. Clin. Invest. 101:1899-1904.

10. Solovey, A., Gui, L., Ramakrishnan, S., Steinberg, M.H., and Hebbel, R.P. 1999. Sickle cell anemia as a possible state of enhanced anti-apoptotic tone: survival effect of vascular endothelial growth factor on circulating and unanchored endothelial cells. Blood. 93:3824-3830.

11. Buchanan, G.R., and Glader, B.E. 1978. Leukocyte counts in children with sickle cell disease. Comparative values in the steady state, vasoocclusive crisis, and bacterial infection. Am. J. Dis. Child. 132:396-398.

12. Boggs, D.R., Hyde, F., and Srodes, C. 1973. An unusual pattern of neutrophil kinetics in sickle cell anemia. Blood. 41:59-65.

13. Francis, R.B.J., and Haywood, L.J. 1992. Elevated immunoreactive tumor necrosis factor and interleukin-1 in sickle cell disease. J. Natl. Med. Assoc. 84:611-615.

14. Moore, C., et al. 1996. Elevated soluble adhesion molecules in sickle hemoglobinopathy patients during the steady state and crisis. Proceedings of the National Sickle Cell Disease Program 21st Annual Meeting. March 6-9, 1996, Mobile, Alabama, USA. p. 8. (Abstr.)

15. Stuart, M.J., and Setty, B.N. 1999. Sickle cell acute chest syndrome: pathogenesis and rationale for treatment. Blood. 94:1555-1560.

16. Oh, S.O., Ibe, B.O., Johnson, C., Kurantsin-Mills, J., and Raj, J.U. 1997. Platelet-activating factor in plasma of patients with sickle cell disease in steady state. J. Lab. Clin. Med. 130:191-196.

17. Sultana, C., Shen, Y., Rattan, V., Johnson, C., and Kalra, V.K. 1998. Interaction of sickle erythrocytes with endothelial cells in the presence of endothelial cell conditioned medium induces oxidant stress leading to transendothelial migration of monocytes. Blood. 92:3924-3935.

18. Barrett-Connor, E. 1971. Bacterial infection and sickle cell anemia. An analysis of 250 infections in 166 patients and a review of the literature. Medicine (Baltimore). 50:97-112.

19. Lachant, N.A., and Oseas, R.S. 1985. Case report: vaso-occlusive crisis associated neutrophil dysfunction in patients with sickle cell disease. Am. J. Med. Sci. 294:253-257.

20. Korthuis, R.J., Grisham, M.B., and Granger, D.N. 1988. Leukocyte depletion attenuates vascular injury in postischemic skeletal muscle. Am.J. Physiol. 254:H823-H827.

21. Mori, E., del Zoppo, G.J., Chambers, J.D., Copeland, B.R., and Arfors, K.E. 1992. Inhibition of polymorphonuclear leukocyte adherence suppresses no-reflow after focal cerebral ischemia in baboons. Stroke. 23:712-718.

22. Welbourn, C.R., et al. 1991. Neutrophil elastase and oxygen radicals: synergism in lung injury after hindlimb ischemia. Am. J. Physiol. 260:H1852-H1856.

23. Winn, R.K., Liggitt, D., Vedder, N.B., Paulson, J.C., and Harlan, J.M. 1993. Anti-P-selectin monoclonal antibody attenuates reperfusion injury to the rabbit ear. J. Clin. Invest. 92:2042-2047.

24. Ma, X.L., Tsao, P.S., and Lefer, A.M. 1991. Antibody to CD-18 exerts endothelial and cardiac protective effects in myocardial ischemia and reperfusion. J. Clin. Invest. 88:1237-1243.

25. McEver, R.P. 1994. Selectins. Curr. Opin. Immunol. 6:75-84.

26. Carlos, T.M., and Harlan, J.M. 1994. Leukocyte-endothelial adhesion molecules. Blood. 84:2068-2101.

27. McEver, R.P. 1997. Selectin-carbohydrate interactions during inflammation and metastasis. Glycoconj. J. 14:585-591.

28. von Andrian, U.H., et al. 1991. Two-step model of leukocyte-endothelial cell interaction in inflammation: distinct roles for LECAM-1 and the leukocyte beta 2 integrins in vivo. Proc. Natl. Acad. Sci. USA. 88:7538-7542.

29. Fabry, M.E., Nagel, R.L., Pachnis, A., Suzuka, S.M., and Costantini, F. 1992. High expression of human beta S- and alpha-globins in transgenic mice: hemoglobin composition and hematological consequences. Proc. Natl. Acad. Sci. USA. 89:12150-12154.

30. Fabry, M.E., et al. 1992. High expression of human beta S- and alphaglobins in transgenic mice: erythrocyte abnormalities, organ damage, and the effect of hypoxia. Proc. Natl. Acad. Sci. USA. 89:12155-12159.

31. Baez, S. 1973. An open cremaster muscle preparation for the study of blood vessels by in vivo microscopy. Microvasc. Res. 5:384-394.

32. Kaul, D.K., Fabry, M.E., Costantini, F., Rubin, E.M., and Nagel, R.L. 1995. In vivo demonstration of red cell-endothelial interaction, sickling and altered microvascular response to oxygen in the sickle transgenic mouse. J. Clin. Invest. 96:2845-2853.

33. Chen, D., and Kaul, D.K. 1994. Rheologic and hemodynamic characteristics of red cells of mouse, rat and human. Biorheology. 31:103-113.

34. Silva, J., and Intaglietta, M. 1974. The correlation of photometric signals derived from in vivo red blood cell flow in microvessels. Microvasc. Res. 7:156-169. 
35. Wayland, H., and Johnson, P.C. 1967. Erythrocyte velocity measurement in microvessels by a two-slit photometric method. J. Appl. Physiol. 22:333-337.

36. Baker, M., and Wayland, H. 1974. On-line volume flow rate and velocity profile measurement for blood in microvessels. Microvasc. Res. 7:131-143.

37. Seki, J., and Lipowsky, H.H. 1989. In vivo and in vitro measurements of red cell velocity under epifluorescence microscopy. Microvasc. Res. 38:110-124.

38. Lipowsky, H.H., Usami, S., and Chien, S. 1980. In vivo measurements of "apparent viscosity" and microvessel hematocrit in the mesentery of the cat. Microvasc. Res. 19:297-319.

39. Dacie, J.V., and Lewis, S.M. 1975. Practical hematology. Churchill Livingstone. Edinburgh, United Kingdom. 47-72.

40. Royall, J.A., and Ischiropoulos, H. 1993. Evaluation of 2',7'-dichlorofluorescin and dihydrorhodamine 123 as fluorescent probes for intracellular $\mathrm{H}_{2} \mathrm{O}_{2}$ in cultured endothelial cells. Arch. Biochem. Biophys. 302:348-355.

41. Kurose, I., et al. 1995. Microvascular responses to inhibition of nitric oxide production. Role of active oxidants. Circ. Res. 76:30-39.

42. Emmendorffer, A., Hecht, M., Lohmann-Matthes, M.L., and Roesler, J. 1990. A fast and easy method to determine the production of reactive oxygen intermediates by human and murine phagocytes using dihydrorhodamine 123. J. Immunol. Methods. 131:269-275.

43. Harris, A.G., Costa, J.J., DeLano, F.A., Zweifach, B.W., and SchmidSchonbein, G.W. 1998. Mechanisms of cell injury in rat mesentery and cremaster muscle. Am. J. Physiol. 274:H1009-H1015.

44. Mayadas, T.N., Johnson, R.C., Rayburn, H., Hynes, R.O., and Wagner, D.D. 1993. Leukocyte rolling and extravasation are severely compromised in P selectin-deficient mice. Cell. 74:541-554.

45. Bullard, D.C., et al. 1996. Infectious susceptibility and severe deficiency of leukocyte rolling and recruitment in E-selectin and P-selectin double mutant mice. J. Exp. Med. 183:2329-2336.

46. Osarogiagbon, U.R., et al. 2000. Reperfusion injury pathophysiology in sickle transgenic mice. Blood. 96:314-320.

47. House, S.D., and Lipowsky, H.H. 1987. Leukocyte-endothelium adhesion: microhemodynamics in mesentery of the cat. Microvasc. Res. 34:363-379.

48. Lipowsky, H.H., and Chien, S. 1989. Role of leukocyte-endothelium adhesion in affecting recovery from ischemic episodes. Ann. NY Acad. Sci. 565:308-315.

49. Kubes, P., Jutila, M., and Payne, D. 1995. Therapeutic potential of inhibiting leukocyte rolling in ischemia/reperfusion. J. Clin. Invest. 95:2510-2519.

50. Jones, S.P., Girod, W.G., Granger, D.N., Palazzo, A.J., and Lefer, D.J. 1999. Reperfusion injury is not affected by blockade of P-selectin in the diabetic mouse heart. Am. J. Physiol. 277:H763-H769.

51. Kanwar, S., Smith, C.W., and Kubes, P. 1998. An absolute requirement for P-selectin in ischemia/reperfusion-induced leukocyte recruitment in cremaster muscle. Microcirculation. 5:281-287.

52. Ley, K. 1995. Gene-targeted mice in leukocyte adhesion research [erratum 1995, 2:301]. Microcirculation. 2:141-150.

53. Solovey, A.A., Solovey, A.N., Harkeness, J., and Hebbel, R.P. 1999. Therapeutic modulation of vascular endothelial cell activation state as therapy for sickle cell disease. Blood. 94:676a. (Abstr.)

54. Bank, N., et al. 1998. Peroxynitrite formation and apoptosis in transgenic sickle cell mouse kidneys. Kidney Int. 54:1520-1528.

55. Kunkel, E.J., et al. 1996. Absence of trauma-induced leukocyte rolling in mice deficient in both P-selectin and intercellular adhesion molecule 1. J. Exp. Med. 183:57-65.

56. Ley, K., et al. 1995. Sequential contribution of L- and P-selectin to leukocyte rolling in vivo. J. Exp. Med. 181:669-675.

57. Paszty, C., et al. 1997. Transgenic knockout mice with exclusively human sickle hemoglobin and sickle cell disease. Science. 278:876-878.

58. Swerlick, R.A., Eckman, J.R., Kumar, A., Jeitler, M., and Wick, T.M. 1993. Alpha 4 beta 1-integrin expression on sickle reticulocytes: vascular cell adhesion molecule-1-dependent binding to endothelium. Blood. 82:1891-1899. 